\title{
Studies of the Kinetics for Rhodium Adsorption onto Gallic Acid Derived Polymer: the Application of Nonlinear Regression Analysis
}

\author{
M. CAN* \\ Vocational School of Arifiye, 54580, Sakarya University, Turkey
}

\begin{abstract}
The kinetic behaviors of solute adsorption at solid/solution interfaces of rhodium (III) complexes from chloride solutions $(1 \mathrm{M} \mathrm{HCl})$ onto gallic acid formaldehyde resol polymer were studied by batch mode. Kinetics was analyzed by the pseudo first-order, the pseudo second-order, the Elovich and the intraparticle diffusion kinetic equations using nonlinear regression analysis. The rate constants of four models were calculated. The Non-linear Chi-square analysis and correlation coefficients have been calculated in order to assess which model provides the best fitting predicted data with experimental results. The pseudo second-order equation provides the best fit to experimental data. The first initial kinetics is governed by the rate of surface reaction. When the adsorbed amount reaches about $95 \%$ of the equilibrium coverage in the first sixty minutes, it switches to another kinetics governed by the rate of intraparticle diffusion. The proposed adsorption of the aquachlororhodium (III) species mostly takes place via a ligand exchange mechanism.
\end{abstract}

DOI: $10.12693 /$ APhysPolA.127.1308

PACS: $82.30 . \mathrm{Hk}$

\section{Introduction}

Due to great demand and their limited ore reserves, platinum group metals (PGM) recovery became important during past decade. As a hydrometallurgical technique, solvent extraction is one of the most efficient ways for large scale operations. In cases of small scale operation, adsorption can be efficiently applied.

In our previous papers, we reported how to successfully synthesize and characterize gallic acid resol polymer (GAR) particles [1], subsequently adsorption isotherm, thermodynamic and elution experiments were performed [2]. The adsorption of rhodium (III) ions onto GAR takes place through the formation of a ligand substituted metal-polymer complex. In this research, the adsorption kinetics behavior of GAR particle toward Rh (III) ions in model solutions, in strongly acidic conditions was investigated batchwise. To investigate the kinetic of Rh (III) interaction, characteristic constants of sorption were determined using a pseudo first- and second-order equation, intraparticle diffusion equation and the Elovich equation, respectively, and compared. Kinetic parameters of experimental data were calculated using nonlinear regression with the help of Microsoft Excel's Solver Extension software program. Chi-square test was used to evaluate the models which have best fit to experimental data.

\section{Experimental \\ 2.1. Materials}

$\mathrm{RhCl}_{3} \cdot 3 \mathrm{H}_{2} \mathrm{O}, \mathrm{NH}_{3}, \mathrm{HCOH}, \mathrm{HNO}_{3}, \mathrm{HCl}, \mathrm{NaCl}$ and $\mathrm{NaOH}$ were purchased from Merck Company. AAS standard solutions for determination of PGM, were purchased

*e-mail: mstfacan@gmail.com

form UltraScientific Company. All other reagents were analytical grade. Preparation of GAR polymer was explained in detail at our recent publication, dealing with synthesis and characterization of polymer [1]. The aqueous $\mathrm{Rh}$ (III) stock solution was prepared from solid $\mathrm{RhCl}_{3} \cdot 3 \mathrm{H}_{2} \mathrm{O}$ in $1.0 \mathrm{M} \mathrm{HCl}$. The Rh (III) solutions were prepared by dilution with ultra-pure water (Milli-Q, UK).

\subsection{Adsorption studies}

The rhodium (III) solution was prepared by diluting the stock solutions to mainly $50 \mathrm{mg} / \mathrm{l}$ concentrations. $50 \mathrm{ml}$ of metal ion solutions were prepared for adsorption experiments and stirred after adding $200 \mathrm{mg}$ GAR particles. All adsorption experiments were carried out in a standard and strictly adhered to batchwise system. Only adsorption capacity experiments were carried out by agitating $1 \mathrm{~g}$ GAR with $1000 \mathrm{ml}$ of metal solution of the various initial metal concentrations, for $150 \mathrm{~min}$ (the time required for equilibrium to be reached between the adsorbed metal ions and metal ions in solution). The experiments were performed at $300 \mathrm{rpm}$. The initial pHs of the solutions were controlled by adding a small amount of $\mathrm{HCl}, \mathrm{HNO}_{3}, \mathrm{NaOH}$ and $\mathrm{HClO}_{4}$. At the end of the adsorption period, $15 \mathrm{ml}$ samples were centrifuged and the solutions were filtered through a $0.45 \mu \mathrm{m}$ Milipore filter paper to avoid any solid particle in the aqueous phase. Samples were measured using AAS. All the adsorption tests were performed at least twice, so as to avoid wrong interpretation owing to any experimental errors. FAAS was calibrated using 0, 4, 12 and 20 ppm standard solution for Rh (III) in $1 \mathrm{M} \mathrm{HCl}$. Samples were diluted to measurement limits for precise results. Amount of adsorbed metal ions was calculated from the concentrations in solutions before and after adsorption process. Results were taken from the average of three scans for each sample. 
The amount of metal adsorbed onto GAR, $q_{e}(\mathrm{mg} / \mathrm{g})$, was calculated as follows:

$$
q_{e}=\left(C_{0}-C_{t}\right) V / W
$$

where $C_{0}$ and $C_{t}$ are the initial and value at time $t$ of liquid-phase concentrations of the metal $(\mathrm{mg} / \mathrm{l})$, respectively. $V$ the volume of the solution (l) and $W$ the weight of the dry GAR used (g). Each experiment was performed at least twice, under identical conditions. Twice tests showed that the standard deviation of the measurement was $\pm 2.5 \%$.

\subsection{Adsorption kinetics}

To simulate the adsorption kinetics, here five commonly used models, pseudo first- and second-order equation, intraparticle diffusion equation and the Elovich equation, were applied for rhodium-GAR interactions. These isotherms can be seen at Table I.
In the industrial usage of adsorbents, the time dependence of adsorption on solid surfaces is named as adsorption kinetics. With the development of the theory of equilibrium of adsorption on heterogeneous solid surfaces, the theory of adsorption-desorption kinetics on heterogeneous surfaces was also developed. Adsorption kinetics is determined by the following stages:

1. Diffusion of molecules from the bulk phase towards the interface space (so-called external diffusion)

2. Diffusion of molecules inside the pores (internal diffusion)

3. Diffusion of molecules in the surface phase (surface diffusion)

4. Adsorption-desorption elementary processes.

Used adsorption kinetic equation.

TABLE I

\begin{tabular}{c|c|c|c|c}
\hline \hline & Equation & Integrated & Constants & Ref. \\
\hline $\begin{array}{c}\text { Lagergren } \\
\text { pseudo first }\end{array}$ & $\frac{\mathrm{d} q_{t}}{\mathrm{~d} t}=k_{1}\left(q_{e}-q_{t}\right)$ & $\log \left(q_{e}-q_{t}\right)=\log q_{e}-\frac{k_{1}}{2.303} t$ & $k_{1}\left(\mathrm{~min}^{-1}\right)$ & {$[5]$} \\
\hline $\begin{array}{c}\text { Pseudo } \\
\text { second-order }\end{array}$ & $\frac{\mathrm{d} q_{t}}{\mathrm{~d} t}=k_{2}\left(q_{e}-q_{t}\right)^{2}$ & $\frac{t}{q_{t}}=\frac{1}{k q_{e}^{2}}+\frac{1}{q_{e}} t$ & $k_{2}\left(\mathrm{~g} \mathrm{mg}^{-1} \mathrm{~min}^{-1}\right)$ & {$[6]$} \\
\hline Elovich & $\frac{\mathrm{d} q_{t}}{\mathrm{~d} t}=\alpha \mathrm{e}^{-\beta q_{t}}$ & $q_{t}=\frac{1}{\beta} \ln (\alpha \beta)+\frac{1}{\beta} \ln t$ & $\begin{array}{c}\alpha\left(\mathrm{mg} \mathrm{g}^{-1} \mathrm{~min}^{-1}\right) \\
\beta\left(\mathrm{mg} \mathrm{g}^{-1}\right)\end{array}$ & {$[7-9]$} \\
\hline $\begin{array}{c}\text { Intraparticle } \\
\text { diffusion }\end{array}$ & $q_{t}=k_{\text {int }} t^{1 / 2}$ & & $k_{\text {int }}\left(\mathrm{mg} \mathrm{g}^{-1} \mathrm{~min}^{-\frac{1}{2}}\right)$ & {$[3]$}
\end{tabular}

In the case of sorption kinetics on micro porous solids, a series of other mechanisms may additionally take place, sui generis. Diffusion in micropores carries the character of activated diffusion which usually, is described by Weber and Morris [3]. Before adsorbing species may enter micropores, the penetration of surface barriers may be necessary. When adsorption systems fit Langmuir isotherm equation, that means adsorbent has non-porous and macroporous character. For non-porous and macroporous solids the internal diffusion may be neglected. In this case, the adsorption kinetics is determined by external diffusion and molecular adsorption-desorption processes. The Langmuirian kinetics, based on the ideal monolayer adsorbed model, proved to be deceptive for most real adsorption systems that include structurally high porous and energetically heterogeneous solids. On the other hand, the adsorption-desorption kinetics theories are technologically extremely important, because the diffusion of adsorbed particles on solid surfaces is a phenomenon of great importance in catalysis, metallurgy, microelectronics, material science and other numerous scientific and technological applications [4].

\subsection{Error analysis}

Linear regression has been one of the most viable tools defining the best-fitting relationship quantifying the distribution of adsorbates, mathematically analyzing the adsorption systems and verifying the consistency and the- oretical assumptions of an isotherm model. Concomitant with the development of computer technology, the progression of the nonlinear isotherm modeling has extensively been facilitated. Contrary to the linearization models, nonlinear regression usually involves the minimization or maximization of error distribution between the experimental data and the predicted isotherm, based on its convergence criteria. In this study two error functions, the coefficient of determination and nonlinear chisquare test have been used for analyzing the adsorption system $[10,11]$.

Nonlinear chi-square test is a statistical tool necessary for the best fit of an adsorption system, obtained by judging the sum squares differences between the experimental and the calculated data, with each squared difference divided by its corresponding value (calculated from the models). Small $\chi^{2}$ value indicates its similarities while a larger value represents the variation of the experimental data.

$$
X^{2}=\sum_{i=1}^{n} \frac{\left(q_{e, \text { calc }}-q_{e, \text { meas }}\right)^{2}}{q_{e, \text { meas }}}
$$

\section{Results and discussions}

The influence of initial rhodium ion concentration was studied and Fig. 1 shows a series of contact time curves with GAR particles. Initial metal concentrations were studied from 17.18 to $88.50 \mathrm{mg}$ Rh (III)/l. Obtained kinetic data have been analyzed using four models. The 
result is shown in Table II. As seen, the sorption capacities at equilibrium, $q_{e}$, increases from 1.8 to $8.36 \mathrm{mg} / \mathrm{g}$ with an increase in the initial Rh (III) concentration from 17.18 to $88.50 \mathrm{mg} / \mathrm{l}$ with a GAR particle dose of $1 \mathrm{~g} / \mathrm{l}$.

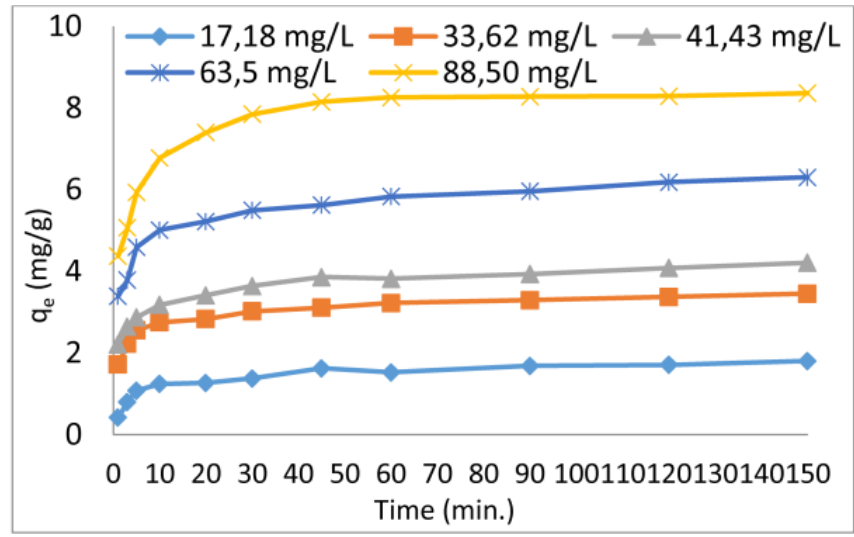

Fig. 1. The effects of contact time and initial $\mathrm{Rh}$ (III) concentration on adsorption $(1 \mathrm{~g}$ GAR, $1 \mathrm{M} \mathrm{HCl}, 293 \mathrm{~K}$, $V=1000 \mathrm{ml}$ ).

Here, pseudo second-order equation's chi-square values were lower than 0.36 . The pseudo second-order equation's calculated equilibrium sorption capacities, $q_{2}$, fit the experimental data. These suggest that the pseudo second-order sorption mechanism is predominant and that the overall rate of the Rh (III) adsorption process appears to be controlled by the chemical process. For the pseudo second-order model, the rate constant, $k_{2}$, increases with an increasing Rh (III) concentration, while the calculated sorption capacity, $q_{2}$, increases with the increase of initial metal concentration (Table II).

Kinetic parameters for sorption of Rh (III)

TABLE II ions onto GAR particles.

\begin{tabular}{|c|c|c|c|c|c|c|c|}
\hline \multirow{2}{*}{$\begin{array}{c}\text { Para- } \\
\text {-meters } \\
(\mathrm{mg} / \mathrm{l})\end{array}$} & \multirow[b]{2}{*}{$\begin{array}{l}q_{e, \exp } \\
(\mathrm{mg} / \mathrm{g})\end{array}$} & \multicolumn{3}{|c|}{$\begin{array}{c}\text { First-order } \\
\text { kinetic equation }\end{array}$} & \multicolumn{3}{|c|}{$\begin{array}{c}\text { Second-order } \\
\text { kinetic equation }\end{array}$} \\
\hline & & $\begin{array}{c}\mathrm{q}_{1} \\
(\mathrm{mg} / \mathrm{g})\end{array}$ & $\begin{array}{c}k_{1} \\
(1 / \min )\end{array}$ & $X^{2}$ & $\begin{array}{c}\mathrm{q}_{2} \\
(\mathrm{mg} / \mathrm{g})\end{array}$ & $\begin{array}{c}k_{2} \\
(\mathrm{~g} / \mathrm{mg} / \mathrm{min})\end{array}$ & $X^{2}$ \\
\hline 15.38 & 1.80 & 1.494 & 0.260 & 0.450 & 1.674 & 1.674 & 0.0685 \\
\hline 30.17 & 3.45 & 2.997 & 0.642 & 0.998 & 3.212 & 3.212 & 0.0916 \\
\hline 37.22 & 4.21 & 3.549 & 0.694 & 2.455 & 3.840 & 3.840 & 0.2085 \\
\hline 57.20 & 6.30 & 5.424 & 0.646 & 5.044 & 5.817 & 5.817 & 0.2868 \\
\hline 80.15 & 36 & 7.644 & 0.490 & 6.623 & 8.133 & 8.133 & 0.3589 \\
\hline \multirow{2}{*}{$\begin{array}{r}\text { Para- } \\
\text {-meters } \\
(\mathrm{mg} / \mathrm{l}) \\
\end{array}$} & \multirow[b]{2}{*}{$\begin{array}{c}q_{e, \exp } \\
(\mathrm{mg} / \mathrm{g}) \\
\end{array}$} & \multicolumn{3}{|c|}{$\begin{array}{c}\text { The Elovich } \\
\text { equation }\end{array}$} & \multicolumn{3}{|c|}{$\begin{array}{c}\text { Intraparticle } \\
\text { diffusion equation }\end{array}$} \\
\hline & & $\begin{array}{c}\alpha \\
(\mathrm{mg} / \mathrm{g} / \mathrm{min}) \\
\end{array}$ & $\begin{array}{c}\beta \\
(\mathrm{mg} / \mathrm{g})\end{array}$ & $X^{2}$ & $(\mathrm{mg} /$ & $\begin{array}{l}\mathrm{k}_{\text {int }} \\
\left.\mathrm{g} / \min ^{1 / 2}\right)\end{array}$ & $X^{2}$ \\
\hline 15.38 & 1.80 & 2.026 & 3.868 & 0.0739 & & 0.197 & 1.645 \\
\hline 30.17 & 3.45 & 102.8 & 3.066 & 0.0323 & & 0.398 & 5.612 \\
\hline 37.22 & 4.21 & 114.3 & 2.535 & 0.0084 & & 0.480 & 6.545 \\
\hline 57.20 & 6.30 & 212.9 & 1.715 & 0.0473 & & 0.724 & 10.16 \\
\hline 80.15 & 8.36 & 126.5 & 1.109 & 0.1501 & & 0.992 & 14.02 \\
\hline
\end{tabular}

A comparison of non-linear calculated and measured results for $88.5 \mathrm{mg} / 1$ initial metal ion concentration is shown in Fig. 2. The pseudo second-order equation provides the best correlation for the sorption process, whereas the first-order and Elovich equations also fit the experimental data well as opposed to each other. The intraparticle equations do not give a good fit to the experimental data for the sorption of Rh (III). The secondorder kinetic model, based on the assumption that the rate limiting step may be chemical sorption or chemisorption involving valence forces through sharing or exchange of electrons between sorbent and sorbate [12].

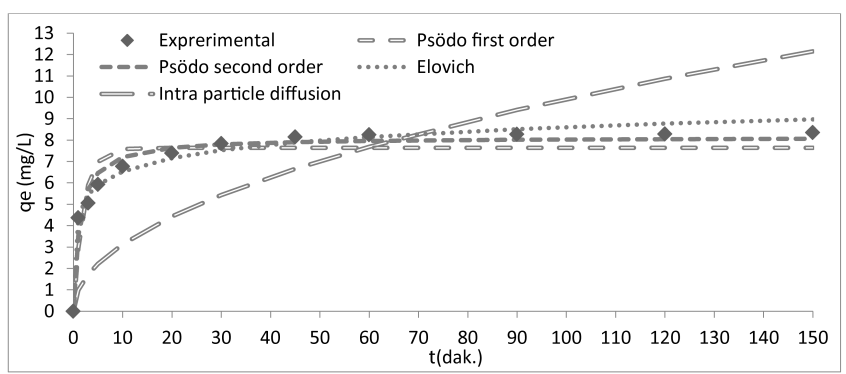

Fig. 2. The measured and non-linear modeled time profiles for adsorption of Rh (III) ions onto GAR particles, at $88.5 \mathrm{mg} / \mathrm{l}$ initial concentration, at temperature of $293{ }^{\circ} \mathrm{K}(1 \mathrm{~g} \mathrm{GAR}, 1 \mathrm{M} \mathrm{HCl}, V=1000 \mathrm{ml})$.

\section{Conclusions}

In this study, the kinetic of removal of $\mathrm{Rh}$ (III) ions from aqueous solution onto GAR particles was investigated in the batch mode. For pseudo first-order, pseudo second-order, the Elovich, and intraparticle diffusion equations, nonlinear regression method was used. Nonlinear method could be a better way to obtain the kinetic parameters.

For all the systems examined, the pseudo second-order kinetic model provided the best correlation of the experimental data.

\section{References}

[1] M. Can, E. Bulut, M. Özacar, Ind. Eng. Chem. 51, 6052 (2012).

[2] M. Can, Acta Phys. Pol. A (2015).

[3] W.J. Weber Jr., J.C. Morris, J. Sanit. Eng. Div. Am. Soc. Civil Eng. 89, 53 (1963).

[4] A. Dabrowski, Adv. Colloid Interface Sci. 93, 135 (2001).

[5] S. Lagergren, Kungliga Svenska Vetenskapsakademiens, Handlingar 24, 1 (1898).

[6] Y.S. Ho, G. McKay, Process Biochem. 34, 451 (1999).

[7] Y. Zeldovich, Acta physicochim. (U.R.S.S.) 1, 449 (1934).

[8] S. Roginskii, Y. Zeldovich, Acta physicochim. (U.R.S.S.) 1, 554 (1934).

[9] S.Y. Elovich, G.RI. Zhabrova, Zhur. Fiz. Khim (U.R.S.S.) 13, 1761 (U.R.S.S.).

[10] K.V. Kumar, S. Sivanesan, J. Hazard. Mater. 123, 288 (2005).

[11] M. Can, Res. J. Chem. Environ. 17, 117 (2013).

[12] M. Can, Desalin. Water Treat., (2015). 